(D) Rosângela Alves Pereira ${ }^{1}$

(1) Mariana Costa Monteiro ${ }^{2}$

(1D)Eliane Lopes Rosado ${ }^{3}$

(DTatiana El-Bacha Porto 3

(D)Ana Luisa Kremer Faller ${ }^{3}$

(1D) Patrícia de Carvalho Padilha ${ }^{3}$

(DE) Elizabeth Accioly ${ }^{3}$

1 Universidade Federal do Rio de Janeiro, Instituto de Nutrição Josué de Castro, Departamento de Nutrição Social e Aplicada. Rio de Janeiro, RJ, Brasil.

2 Universidade Federal do Rio de Janeiro, Instituto de Nutrição Josué de Castro, Departamento de Nutrição Básica e

Experimental. Rio de Janeiro, RJ, Brasil.

${ }^{3}$ Universidade Federal do Rio de Janeiro, Instituto de Nutrição Josué de Castro Departamento de Nutrição e Dietética. Rio de Janeiro, RJ, Brasil.

Correspondência

Rosângela Alves Pereira

roapereira@gmail.com

\section{Trajetória, avanços e perspectivas da pós- graduação no Instituto de Nutrição Josué de Castro, Universidade Federal do Rio de Janeiro}

\author{
History, advances, and perspectives of graduate studies at \\ Instituto de Nutrição Josué de Castro, Universidade Federal \\ do Rio de Janeiro
}

\section{Resumo}

O Instituto de Nutrição Josué de Castro (INJC), da Universidade Federal do Rio de Janeiro (UFRJ), foi criado em 1946 como instituição de ensino e pesquisa na área de Nutrição. Este trabalho, submetido na categoria "Perspectivas", descreve a trajetória do ensino de Pós-Graduação (PG) no INJC, no ano do jubileu dos 75 anos do INJC. Para elaborar este relato, foram consultados artigos, livros, documentos, relatórios, atas e anotações do INJC e da UFRJ. O ensino de PG no INJC teve início no final da década de 1970, com a oferta de dois cursos de especialização. Em 1985, foi implantado o mestrado em Nutrição Humana. Atualmente, o INJC é a única instituição da área de Nutrição no Brasil que abriga um programa acadêmico de mestrado e doutorado, o Programa de Pós-Graduação em Nutrição, e um programa de mestrado profissional, o Programa de Pós-Graduação em Nutrição Clínica. A instituição oferece, ainda, seis cursos de especialização e integra quatro programas de residência multiprofissional da UFRJ. Indicadores favoráveis consistentes expressam o sucesso do esforço coordenado de docentes e discentes dos Programas de PG em iniciativas que privilegiam a obtenção de financiamento, o desenvolvimento de projetos interdisciplinares intra e interinstitucionais e o incentivo à produção acadêmica de qualidade. A pós-graduação no INJC articula-se com o ensino de graduação e com a extensão, contribuindo para a formação qualificada de recursos humanos no campo da Alimentação e Nutrição. Aos 75 anos, o INJC, por meio do ensino de PG, reitera o papel social e acadêmico da universidade pública brasileira.

Palavras-chave: Programas de Pós-Graduação em Saúde. Ciências Nutricionais. Ciência, Tecnologia e Sociedade.

\footnotetext{
Abstract

The Instituto de Nutrição Josué de Castro (Josué de Castro Nutrition Institute - INJC), at the Universidade Federal do Rio de Janeiro (Federal University of Rio de Janeiro - UFRJ) was created in 1946 as an education and research institution in the field of nutrition. This work, presented in the Perspectives modality, describes the history of graduate education (GE) at INJC, in the year of INJC'S 75th anniversary. This work is based on articles, books, documents, reports, minutes, and notes from UFRJ and INJC's Graduate Programs. GE at INJC began in the late 1970s, with the offer of specialization courses. In 1985, the Master's Degree Program in Human Nutrition was implemented. Currently, INJC is the only institution in the field of Nutrition in Brazil that hosts academic master's and doctoral programs, the Graduate Program in Nutrition, and a professional master's program, the Graduate Program in Clinical Nutrition. The
} 
institution also offers six specialization courses and integrates four multi-professional internship programs within UFRJ. Consistent favorable indicators express the success of the coordinated efforts of faculty and students of GE Programs in initiatives that prioritize attraction of funding, the implementation of intra- and inter-institutional interdisciplinary projects, and qualified academic production. Graduate education at INJC is linked to undergraduate education and to university outreach programs, contributing to high-quality education and training of human resources in the field of food and nutrition. After 75 years, INJC, through graduate education, reaffirms the social and academic role of Brazilian public universities.

Keywords: Health Postgraduate Programs. Nutritional Sciences. Science Technology and Society. 


\section{INTRODUÇÃO}

O Instituto de Nutrição da Universidade Federal do Rio de Janeiro (UFRJ) foi criado em 1946, a partir da incorporação do Instituto de Tecnologia Alimentar à Universidade do Brasil, com o compromisso de estabelecer uma instituição de ensino e pesquisa na área de Nutrição. ${ }^{1}$ Em 1996, ao completar 50 anos, o Instituto de Nutrição incorporou o nome do seu fundador e primeiro diretor, passando a chamar-se Instituto de Nutrição Josué de Castro (INJC). Desde sua origem, portanto, o INJC vem se pautando por sua vocação para a pesquisa. Neste ano de 2021, por ocasião das comemorações dos seus 75 anos e como um exercício necessário de memória e resgate histórico, apresentamos este trabalho, que teve por objetivo descrever a trajetória do ensino de pós-graduação no INJC.

Para elaborar este relato, foram consultados artigos, livros, documentos, relatórios do sistema da CAPES (Coordenação de Aperfeiçoamento de Pessoal de Nível Superior, Ministério da Educação)a, atas e anotações do acervo documental dos programas de pós-graduação (PPG) do INJC e do Sistema Integrado de Gestão Acadêmica (SIGA) da UFRJ.

O ensino de pós-graduação no INJC teve início no final da década de 1970, quando foram oferecidos os cursos de pós-graduação lato sensu de Especialização em Nutrição Clínica e de Especialização em Nutrição Materno-Infantil,2 os quais formaram o núcleo que levou à criação do Mestrado em Nutrição Humana, em meados dos anos 1980. Esse mestrado englobava as áreas de concentração em Nutrição em Saúde Pública, Ciências dos Alimentos e Nutrição e Dietética e foi o segundo curso de mestrado em Nutrição no Brasil e o primeiro no Estado do Rio de Janeiro. ${ }^{3}$

Atualmente, o INJC abriga o Programa de Pós-Graduação em Nutrição, o Programa de Pós-Graduação em Nutrição Clínica e seis cursos de especialização lato sensu, além de integrar quatro programas de residência vinculados a diferentes unidades hospitalares da UFRJ. Dessa forma, o INJC é a única instituição da área de Nutrição no Brasil que sedia programa acadêmico de mestrado e doutorado e programa de mestrado profissional.

No INJC, os PPGs obedecem às regulamentações da Pró-reitoria de Pós-Graduação e Pesquisa da UFRJ quanto à estruturação acadêmico-administrativa, sendo dirigidos por um(a) coordenador(a) e um(a) vice-coordenador(a), ambos(as) eleitos(as) pelo segmento da comunidade interna vinculada aos PPGs. Os programas têm como instâncias decisórias os respectivos Conselhos Deliberativos, que são compostos por representantes dos docentes e discentes dos respectivos PPGs. Anualmente, como forma de ampliar a participação do corpo social do INJC, são realizados fóruns para debate de temas de interesse dos programas. Nessas ocasiões participam, além de docentes, técnicos e discentes vinculados aos PPGs, os demais membros do corpo social da instituição e convidados externos.

A seguir, são apresentados os principais marcos que contextualizam a trajetória da pós-graduação no INJC.

\section{PROGRAMA DE PÓS-GRADUAÇÃO EM NUTRIÇÃO}

O Programa de Pós-graduação em Nutrição (PPGN) da UFR oferece os cursos de mestrado e doutorado acadêmicos, cuja missão é formar mestres e doutores com pensamento crítico para atuarem em atividades acadêmicas e científicas no ensino, na pesquisa e na área técnica no campo da Alimentação e Nutrição.

\footnotetext{
a Informações gerais sobre os programas de pós-graduação do INJC em: Coordenação de Aperfeiçoamento de Pessoal de Nível Superior. Plataforma Sucupira [Internet]. Brasília: CAPES; c2016 [cited 2021 Jul 11]. Disponível em: https://sucupira.capes.gov.br/sucupira/public/consultas/coleta/programa/quantitativos/quantitativoBuscaAvancada.jsf;j sessionid=FHj807fSxH3PndNnEAZSvoUq.sucupira-215
} 
A criação do PPGN se deu em 1985, com a implantação do curso de Mestrado em Nutrição Humana, a partir do convênio entre o INJC e a agência de cooperação técnica alemã GTZb , sendo o primeiro mestrado em Nutrição no estado do Rio de Janeiro. No início dos anos 2000, o PPGN passou por um processo de reformulação e, desde então, tem evoluído de maneira consistente e positiva, de modo que os indicadores que medem seu desempenho têm apresentado contínua evolução. Em 2006, foi implantado o curso de Doutorado em Ciências Nutricionais, também o primeiro doutorado em Nutrição do estado do Rio de Janeiro. Com a permanente evolução dos indicadores de desempenho do programa, resultado do esforço conjunto dos corpos docente e discente, o PPGN alcançou o conceito 6 em 2017, e, atualmente está entre os três programas de pós-graduação com maior conceito na área de Nutrição no Brasil.

No momento, 17 docentes permanentes e quatro colaboradores integram o quadro de orientadores credenciados no PPGN, que atuam em quatro linhas de pesquisa: Bioquímica Nutricional, Ciência e Tecnologia dos Alimentos, Epidemiologia Nutricional e Micronutrientes. A formação acadêmica do corpo docente é diversificada e atende às especificidades das linhas de pesquisa, tendo a maioria dos docentes realizado estágio pós-doutoral, missões e/ou cursos de capacitação no exterior, favorecendo a internacionalização do programa.

A estrutura curricular do PPGN é desenhada para promover a formação de recursos humanos com capacidade de articulação do pensamento científico e com ampla visão da pesquisa científica aplicada às questões da sociedade. As disciplinas obrigatórias dos cursos de mestrado e doutorado favorecem e estimulam o diálogo e a troca de experiências entre discentes e docentes das diferentes linhas de pesquisa do programa, promovendo a formação em caráter interdisciplinar. No mestrado, as disciplinas obrigatórias totalizam 285 horas, e no doutorado, 405 horas. Essas disciplinas abordam conteúdos voltados para: (i) a fundamentação teórica e metodológica, atualização de conteúdos de ética e bioética, e análise e gestão de dados no âmbito da pesquisa científica; (ii) o fornecimento de ferramentas de redação científica; (iii) o suporte à formação didático-pedagógica; e (iv) o debate científico no âmbito da pesquisa no campo da Nutrição. Para integralização da carga horária de disciplinas, os discentes dos cursos de mestrado e doutorado precisam cumprir, no mínimo, 75 h e 135 h de disciplinas eletivas, respectivamente, que permitem a complementação necessária à formação do estudante.

Até agosto de 2021 o PPGN titulou 388 mestres e 100 doutores e, atualmente, possui 86 discentes matriculados, sendo 31 de mestrado e 55 de doutorado. Destaca-se, também, a forte inserção de recém-doutores no PPGN, demonstrando a elevada capacidade de nucleação de jovens doutores, havendo em atuação, no momento, 20 pós-doutorandos.

Os principais produtos gerados das dissertações e teses defendidas no âmbito do PPGN incluem publicações em periódicos nacionais e internacionais, livros e capítulos de livros. No último quadriênio (2017-2020) de avaliação dos PPGs pela CAPES, o PPGN publicou 307 artigos em periódicos, dos quais 48\% em periódicos Qualis` A1 e A2 da CAPES, e 66\% em periódicos nos estratos A1, A2 e B1, sendo que cerca de 20\% incluíram pesquisadores internacionais entre os coautores. Entre 2017 e 2020, houve aumento de cerca de 30\% no número de artigos publicados pelo PPGN, enquanto que as publicações nos estratos Qualis A1 e A2 aumentaram em quase 40\%. Os discentes participaram da autoria de $70 \%$ dos artigos produzidos no programa ao longo dos últimos quatro anos, enquanto a participação discente nos artigos publicados em periódicos Qualis A1 e A2 aumentou de 26\% para 38\%. Dos 60 livros e capítulos de livros produzidos no quadriênio, 38\% contaram com a participação de discentes e/ou egressos do programa, $10 \%$ possuem coautoria de pesquisador estrangeiro, 17\% foram publicados por editoras

\footnotetext{
${ }^{\mathrm{b}}$ GTZ: Deutsche Gesellschaft für Technische Zusammenarbeit [Agência Alemã de Cooperação Técnica], atualmente GIZ Deutsche Gesellschaft für Internationale Zusammenarbeit [Agência Alemã de Cooperação Internacional] (https://www.giz.de/en/html/index.html)

c a classificação dos periódicos pela CAPES está disponível na Plataforma Sucupira em https://sucupira.capes.gov.br/sucupira/public/consultas/coleta/veiculoPublicacaoQualis/listaConsultaGeralPeriodicos.jsf
} 
universitárias e 70\% foram submetidos à revisão por pares. Tais indicadores demonstram a continuidade da evolução do PPGN no âmbito da pesquisa no campo da Nutrição.

\section{PROGRAMA DE PÓS-GRADUAÇÃO EM NUTRIÇÃO CLÍNICA}

Em 2012 o INJC propôs a criação de um mestrado profissional visando atender as demandas da profissão, do mercado de trabalho e da sociedade. Foi então criado o Mestrado Profissional do Programa de Pós-graduação em Nutrição Clínica (PPGNC), um dos quatro programas de mestrado profissional da área de Nutrição em atuação no país, tendo sido o primeiro a ser implantado em instituição pública de ensino superior.

A proposta desse mestrado profissional veio atender a demanda reprimida de profissionais da área de Nutrição Clínica interessados em aperfeiçoamento profissional em nível de mestrado e de exigências do mercado de trabalho por profissionais com visão crítica e inovadora que propiciem a transformação do local de atuação. Àquela altura, não havia cursos de mestrado profissional com foco em Nutrição Clínica no Brasil e considerava-se imperativa a necessidade de consolidação dessa área de atuação dentro dos PPGs no país. Dessa forma, com o PPGNC, foi criado um ambiente stricto sensu pioneiro para qualificação técnica e científica dos profissionais que atuavam na Nutrição Clínica, agregando inovação científica para o mercado de trabalho, com duas linhas de pesquisa: Alimentos e Produtos Nutricionais com Aplicação em Nutrição Clínica e Processos e Protocolos em Nutrição Clínica, as quais abordam de forma abrangente o tratamento clínico-nutricional. Consistentemente com o perfil exigido pela CAPES para essa modalidade, o PPGNC oferece disciplinas específicas, cujo conteúdo programático atende às linhas de pesquisa do Programa e a demanda da área de Nutrição Clínica.

O Mestrado Profissional em Nutrição Clínica do PPGNC tem carga horária total de 360 horas, das quais 270 correspondem a disciplinas obrigatórias. Até junho de 2021, o PPGNC titulou 58 mestres e conta, atualmente, com 19 discentes matriculados e 15 docentes credenciados, sendo 11 permanentes e quatro colaboradores.

A produção acadêmica do PPGNC inclui artigos em periódicos nacionais e internacionais, livros e capítulos de livros e produtos técnicos/tecnológicos, que incluem, por exemplo, material didático instrucional, curso de formação profissional, organização de eventos científicos e relatórios técnico-científicos.

No ano de 2021, o PPGNC passará pelo segundo processo avaliativo na área de Nutrição da CAPES, referente ao quadriênio 2017-2020, quando o número de artigos publicados anualmente mais do que triplicou, passando de 14, no primeiro ano, para 47 no último ano do quadriênio. O mais importante é que a proporção de artigos em periódicos Qualis A1 e A2 sextuplicou, passando de 7\%, em 2017, para 47\% do total de artigos publicados em 2020. Além disso, nesse quadriênio, a inclusão de discentes na produção científica do PPGNC aumentou consideravelmente.

\section{PÓS-GRADUAÇÃO LATO SENSU}

A pós-graduação lato sensu é uma estratégia mais flexível para atender às demandas da inovação de processos e técnicas e de novos campos de atuação, permitindo estreitar a relação entre a academia e a sociedade. Assim, o INJC mantém a tradição de oferta de cursos lato sensu, em consonância com as normas estabelecidas pela UFRJ para o ensino de graduados. Na atualidade, os cursos são custeados pelos próprios alunos, com geração de recursos para garantir sua oferta e expansão, em consonância com as normas regulatórias vigentes da Pró-reitoria de Ensino para Graduados da UFRJ.

Além dos dois cursos de pós-graduação lato sensu iniciais já mencionados, em 1994 foi oferecida a primeira turma do Curso de Especialização em Intervenções Nutricionais em Saúde Coletiva, em 1998, o Curso de 
Especialização em Alimentação Institucional: Ênfase em Planejamento e Controle e, em 2000, o Curso de Especialização em Nutrição em Obstetrícia e Pediatria. Todos esses cursos tiveram um período delimitado de oferta.

Em 2003 foi implantado o Curso de Especialização em Nutrição Clínica (CENC), o de mais longa duração no INJC e vigente até os dias atuais. Esse curso oferta turmas em outros municípios do estado do Rio de Janeiro, tendo já possibilitado a capacitação de mais de 700 nutricionistas.

A partir de 2019, foram implantados novos cursos de especialização no INJC, sendo eles: Fitoterapia Aplicada à Clínica (CEFITO), Nutrição Funcional Aplicada à Clínica (CENUF), Alimentação Coletiva (CEAC), Terapia Nutricional em Pediatria (TENUTPED) e, Terapia Nutricional (CETNUT). Em conjunto, esses cursos disponibilizam mais de 600 vagas anuais e possibilitam a capacitação em diferentes cenários de atuação no campo da Alimentação e Nutrição. No momento está em fase de tramitação o processo para criação do curso de Especialização em Bebidas e Gastronomia, a primeira iniciativa em nível de pós-graduação na UFRJ na área de Gastronomia, que constitui o segundo curso de graduação oferecido pelo INJC.

A necessidade de oferecer treinamento em serviço ensejou a criação de programas de residências multiprofissionais, em cooperação com as unidades hospitalares da UFRJ e outras unidades da área da saúde e das ciências humanas. São elas: Residência Integrada Multiprofissional em Saúde, no Hospital Universitário Clementino Fraga Filho; Residência Multiprofissional em Saúde da Família e Comunidade, no Hospital Escola São Francisco de Assis; Residência Multiprofissional em Saúde Perinatal, na Maternidade Escola; e Residência em Saúde da Criança e do Adolescente, no Instituto de Puericultura e Pediatria Martagão Gesteira.

As diferentes modalidades de pós-graduação lato sensu constituem uma intersecção entre diversos campos de prática e ensino com a pesquisa acadêmica, muitas vezes despertando talentos interessados na pós-graduação stricto sensu. Ademais, a elaboração de monografias de conclusão de curso é um campo de fértil para estudos-pilotos que muitas vezes se expandem e se aperfeiçoam numa dissertação de mestrado. Docentes dos programas stricto sensu também participam dos cursos lato sensu, consolidando o caráter integrador da pós-graduação no INJC.

\section{IMPACTO ACADÊMICO E SOCIAL DA PÓS-GRADUAÇÃO NO INJC}

Os PPGs do INJC apresentam excelente grau de inserção social, a começar pelo processo seletivo discente, alinhado à proposta da UFR de aproximação da instituição com a sociedade de maneira ampla e diversa. Assim, os PPGs oferecem vagas que favorecem ações afirmativas, em especial, para pessoas autodeclaradas pretas, pardas e indígenas e pessoas com deficiência, nos termos da legislação.

Ambos os PPGs buscam seguir as diretrizes do Plano de Desenvolvimento Institucional da UFRJ, estimulando o corpo docente e discente à maior proximidade com a sociedade. Os PPGs têm organizado eventos com o objetivo de ampliar os conhecimentos gerados e agregar participantes externos aos programas. Além disso, docentes e discentes participam ativamente dos eventos acadêmicos e científicos no âmbito do INJC e da UFRJ, bem como em eventos de abrangência local, regional, nacional e internacional, que atingem público ampliado. Essa participação ocorre em diversos espaços, seja como autores, membros das comissões científicas e organizadoras e avaliadores de trabalhos. Particularmente no PPGNC, tem sido estimulada a produção técnica/tecnológica com perspectiva de aplicação na prática em nutrição nas diferentes instituições públicas e privadas.

Nos últimos anos a visibilidade dos PPGs do INJC tem recebido atenção especial, com investimentos no aperfeiçoamento e atualização dos canais de comunicação com o público externo. Tais estratégias objetivam divulgar as informações sobre os programas, suas linhas de trabalho, processos seletivos e outras informações de interesse, estando disponíveis nas páginas de internet próprias de cada programa (http://www.ppgn.ufrj.br; http://www.ppgnc.injc.ufrj.br). Tanto docentes como discentes dos PPGs têm buscado inserção nas redes sociais em 
atividades de divulgação científica e disseminação do conhecimento científico por meio de iniciativas em redes sociais, como perfis no Instagram, Facebook ${ }^{d}$ e no canal de YouTube do INJC (https://www.youtube.com/channel/UCICXd3h32wXCQh0DiB-J1fQ).

Outra forma de inserção social e retorno do conhecimento científico para a sociedade se revela por meio das parcerias com órgãos da UFRJ, instituições governamentais e não governamentais, por meio da participação em projetos de pesquisa, estudos multicêntricos, cursos e consultorias, entre outras atividades. Ademais, a atuação dos egressos dos PPGs vem se pautando por ações que contribuem para a melhoria da gestão pública e privada em nutrição e saúde e para a redução das desigualdades sociais. Os egressos de ambos PPGs atuam como docentes em instituições de ensino superior público e privado e em cursos de pós-graduação lato sensu. Egressos do PPGN ocupam posições importantes na área da pesquisa e do ensino de pós-graduação stricto sensu, como docentes, coordenadores de curso e pesquisadores em instituições de pesquisa e em setores e órgãos da administração pública.

Confirmando sua vocação para o aperfeiçoamento dos nutricionistas que atuam na prática clínica, a maioria dos egressos do PPGNC atua na assistência nutricional em instituições públicas vinculadas ao Sistema Único de Saúde, em diversas regiões do Brasil, enquanto alguns desses egressos deram continuidade à formação acadêmica, ingressando no doutorado. Em enquete realizada pela coordenação do programa, os egressos do PPGNC revelaram que o mestrado propiciou melhor capacitação para atuar profissionalmente na assistência, ensino, pesquisa e extensão. A atuação dos egressos dos PPGs do INJC evidencia seu potencial de nucleação e importante papel na formação de recursos humanos capazes de contribuir para o fortalecimento de outros PPGs e para a formação de novos grupos de pesquisa.

No INJC, OS PPGs se articulam com o ensino de graduação, tanto pela participação dos docentes como responsáveis ou colaboradores em disciplinas da graduação, como pela supervisão de pós-graduandos em seus estágios de docência. Docentes dos PPGs coordenam e participam de ações extensionistas que integram alunos de graduação e pós-graduação, cujas linhas de trabalho se articulam com demandas sociais em distintas interfaces da ciência da Nutrição com outras áreas do saber, especialmente com a Gastronomia. As atividades que se inserem nesse contexto envolvem participações dos pós-graduandos e graduandos em ações de educação sobre alimentação saudável em escolas, vilas olímpicas, praças públicas e museus; acompanhamento e orientações nutricionais a grupos populacionais específicos, como indivíduos com obesidade, crianças e gestantes com diabetes mellitus, atletas vinculados a diferentes organizações e outros grupos em vulnerabilidade social. A integração dos PPGs do INJC com a graduação favorece o nexo da pós-graduação com a extensão e promove a iniciação científica dos graduandos, fortalecendo a formação interdisciplinar de graduandos e pós-graduandos. Tal articulação tem motivado muitos estudantes de graduação a trilhar a senda acadêmica, ingressando nos cursos de mestrado, residências e cursos de especialização.

As atividades do PPGN e do PPGNC extrapolam as fronteiras nacionais e se concretizam em parcerias com universidades e institutos de pesquisa de renome internacional. Atualmente os PPGs mantêm convênios de cooperação acadêmica e científica com mais de 20 instituições de países como os Estados Unidos, França, Espanha, Dinamarca, Holanda, Reino Unido, Austrália, Canadá e Colômbia. Essas colaborações aumentam as possibilidades de cooperação científica em nível internacional, incentivam a capacitação docente e discente, e contribuem para o aprimoramento da pesquisa e da produção acadêmica nos programas.

\footnotetext{
d Instagram: @gisan.ufrj; @crianca_verde_legal; @nutricaomaternaeinfantil_gpsmi, @ladige.ufrj

Facebook: https://www.facebook.com/saudematernaeinfantil
} 


\section{DESAFIOS E PERSPECTIVAS PARA A PÓS-GRADUAÇÃO NO INJC}

As limitações impostas pela pandemia da Covid-19 conduziram a mudanças e adequações para garantir a continuidade das atividades na pós-graduação. O PPGN, o PPGNC e os cursos lato sensu adotaram o ensino remoto na ministração das disciplinas, logrando manter o calendário escolar previsto para 2020 e 2021. A modalidade de trabalho virtual também permitiu a continuidade de instalação de bancas de qualificação e defesa dos trabalhos finais dos cursos e dos processos seletivos para ingresso de alunos nas turmas de 2020 e 2021.

Apesar dos aspectos negativos impostos pela pandemia, o ensino remoto na pós-graduação proporcionou a oportunidade de maior interação com docentes e discentes de outros PPGs do Brasil e mesmo de outros países. Nesse contexto, houve intensa participação de docentes de PPGs de todo o país como convidados em disciplinas e como membros de bancas de qualificação e defesa de teses e dissertações dos nossos PPGs. Mais relevante ainda, tem sido o intercâmbio entre PPGs, com a efetiva participação de discentes de diversas regiões do país em disciplinas oferecidas nos dois programas do INJC e de pós-graduandos do INJC em disciplinas de outros programas.

Ainda em decorrência da pandemia de Covid-19, o PPGN e o PPGNC estão implementando a Plataforma Josué de Castro de Recursos Educacionais Digitais, que visa possibilitar a disseminação de recursos, funcionalidades, ferramentas e conteúdos em formato digital que permitam a interação entre os diferentes atores da universidade e contemplem diversos fins educacionais, sobretudo aqueles relacionados às práticas pedagógicas. Esta Plataforma também se propõe a fomentar a produção de vídeos educativos voltados às aulas práticas, pela criação de um laboratório educacional digital, atendendo a demandas em disciplinas com atividades práticas. Tais estratégias reforçam a integração da pós-graduação e da graduação nas atividades de ensino, pesquisa e extensão.

Os PPGs utilizam o complexo laboratorial do INJC, que é constituído por laboratórios de pesquisa básica e aplicada, laboratório de informática, grupos de pesquisa interinstitucionais e observatório de pesquisa, todos de caráter multiusuário que dão suporte à realização de estudos colaborativos inter- e intra-institucionais, assim como ao ensino na graduação e na pós-graduação, fortalecendo a integração das atividades-fim do INJC.

Nos últimos processos avaliativos na área de Nutrição da CAPES, o PPGN vem superando os aspectos críticos, e a manutenção da excelência é a perspectiva do PPGN e, também, seu principal desafio.

As estratégias do PPGNC para o aprimoramento do programa se concentram na obtenção de financiamento, na promoção de projetos inovadores interdisciplinares e no fortalecimento de parcerias nacionais e internacionais, inclusive com interface com a educação básica.

No atual cenário de negacionismo científico, subfinanciamento da universidade pública e da ciência brasileira ${ }^{4,5}$ é um grande desafio manter a titulação de mestres e doutores, a captação do financiamento para a pesquisa científica, a publicação em periódicos de alto impacto e a colaboração com instituições de pesquisa nacionais e internacionais. As perspectivas dos PPGs do INJC, portanto, são de manutenção do esforço, empenho e resiliência por parte de docentes, discentes e técnicos na construção da ciência na área de Nutrição. Aos 75 anos de existência, o INJC, por meio dos seus programas de pós-graduação, reitera o papel social e acadêmico da universidade brasileira, com inovação e compromisso com a ciência.

\section{REFERÊNCIAS}

1. Brasil. Decreto-Lei No 8.684, de 16 de janeiro de 1946. Autoriza a Universidade do Brasil a incorporar o Instituto de Tecnologia Alimentar. Diário Oficial da União, 1946 Jan 17. Seção 1: 765.

2. Associação Brasileira de Nutrição (ABN). Histórico do nutricionista no Brasil, 1939-1989: coletânea de depoimentos e documentos. Rio de Janeiro: Editora Atheneu, 1991, 442 p. 
3. Kac G, Proença RPC, Prado SD. A criação da área "Nutrição" na Capes. Rev Nutr. 2011; 24 (6): 905-916. DOI: 10.1590/S1415-52732011000600011.

4. Aragão DSS, Souza AMC, Silva SA, Jucá SCS. Cenário político e contribuições da mídia no processo de desvalorização da Ciência no Brasil. RSD [Internet]. 2020 [citado 2021 Jul 11]; 9(9):e371997138. Disponível em: https://rsdjournal.org/index.php/rsd/article/view/7138. DOI: 10.33448/rsd-v9i9.7138

5. Assunção Y. O Cenário da Ciência, Tecnologia e Inovação no Brasil. 2020 Mai 8 [citado 2021 Jul 11]. In:

Observatório da Comunicação Pública da Ciência [Internet]. Belo Horizonte: Pensar a Educação, Pensar o Brasil. [citado 2021 Jul 07]; Disponível em: https: //pensaraeducacao.com.br/observatorio/2020/05/08/o-cenario-daciencia-tecnologia-e-inovacao-no-brasil/.

\section{Colaboradoras}

Pereira RA contribuiu na concepção, revisão, formatação e aprovação da versão final; Monteiro MC, Rosado EL, Porto T El-Bacha, Faller ALK, Padilha PC e Accioly E contribuíram na consulta documental, concepção, redação e revisão final do manuscrito.

Conflito de Interesses: As autoras declaram não haver conflito de interesses.

Recebido: 20 de julho de 2021

Aceito: 27 de julho de 2021 